\title{
Las publicaciones en endocrinología
}

L as publicaciones en medicina y en general en ciencias aumentan y son cada vez más importantes y exigentes en el mundo, y de la calidad de éstas depende que los investigadores quieran enviar sus resultados.

En endocrinología son muy pocas las revistas publicadas en América Latina, y Colombia tuvo el privilegio de tener una de muy buena calidad, la cual se publicó hasta la década de los ochenta, afortunadamente en este momento, con el esfuerzo mancomunado de las directivas de nuestra asociación y del comité editorial, estamos haciendo de nuevo nuestra aparición dentro del mundo de las publicaciones médicas colombianas. Esperamos que la revista de la ACE sea cada vez más importante y logremos acceder a una excelente calificación por parte de los índices nacionales e internacionales y en la opinión de todos nuestros colegas, tarea que dependerá de todos nosotros.

La endocrinología, rama de la medicina y de la biología, ha llegado a un desarrollo de importantes proporciones, especialmente en lo referente a la fisiología y la biología molecular, constituyéndose en la ciencia que estudia los mecanismos que controlan todo el medio interno, por lo cual no sólo reviste interés para los médicos, sino también para todos los científicos dedicados a las ciencias básicas.

Los principales motivos por los cuales los pacientes acuden al endocrinólogo son la obesidad, la diabetes y los trastornos tiroideos, el resto de las alteraciones endocrinas son de baja incidencia y en algunos casos constituyen, porque no decirlo, curiosidades médicas. Este grupo de patologías tiene una incidencia alta dentro de las morbilidades más comunes, además de que en la mayoría de los casos hacen parte de las enfermedades crónicas y en algunos casos silenciosas, las cuales son características del mundo contemporáneo y juegan un papel determinador de la calidad de vida de la población.

En este número de la revista ACE queremos destacar los artículos: Descripción de los hallazgos histopatológicos en la terapia inicial del cáncer de tiroides en un centro de referencia en Bucaramanga, escrito por Juan Carlos Rojas y colaboradores de la Clínica Carlos Ardila Lülle de dicha ciudad ${ }^{(1)}$ y, Presente y futuro del trasplante de islotes pancreáticos. Un tratamiento innovador para la diabetes tipo $1^{(2)}$, además de la presentación de un caso clínico: Primer trasplante de islotes realizado en Colombia. Experiencia Fundación Valle del Lilli ${ }^{(3)}$, en el cual se aplicó la técnica descrita, escrito por Gabriel Jaime Echeverri y colaboradores.

El cáncer diferenciado de tiroides representa aproximadamente el 2\% de todos los cánceres diagnosticados en los Estados Unidos. La incidencia entre los años 2004 y 2008 fue de 5,5 por 100.000 en hombres y de 16,3 por 100.000 en mujeres. La Sociedad Americana de Cáncer estima que 56.460 casos nuevos de cáncer de tiroides fueron diagnosticados en 2012, incluyendo 43.210 mujeres y 13.250 hombres, con 1.780 muertes, para una mortalidad global del 3\%. Es el cáncer más común en mujeres en los Estados Unidos. En Colombia, de acuerdo con los datos publicados por Globocan en el 2000, la tasa ajustada de incidencia de cáncer de tiroides para hombres fue de 1 por 100.000 habitantes y en mujeres, de 3,7 por 100.000 habitantes. De acuerdo con los datos del Instituto Nacional de Cancerología, representa la séptima neoplasia más frecuentemente diagnosticada. Para el año 2001, se presentaron 30 casos incidentes en hombres y 110 casos en mujeres, de los cuales el 90\% fueron carcinomas bien diferenciados de tipo papilar ${ }^{(4)}$.

El trabajo realizado por Rojas es un estudio descriptivo transversal en el cual analizaron los informes de patología de los pacientes intervenidos con diagnóstico de carcinoma de tiroides en el periodo comprendido entre los años 2001 y 2013 en la Clínica Carlos Ardila Lulle de Bucaramanga. Evaluaron 268 piezas quirúrgicas, encontrándose 247 casos de carcinoma papilar, seguido de 16 registros de folicular, 5 de medular y 5 de anaplásico y un solo caso de linfoma de tiroides. Cabe anotar que la proporción y distribución de dicha neoplasia en este grupo por lo demás importante, está muy de acuerdo con la distribución y frecuencia de lo reportado en la literatura internacional. Además, debemos enfatizar que este tipo de neoplasia es asintomática en la mayoría de los casos y que su aparición en los hombres deberá ser motivo de alerta, además de que su pronóstico es favorable en la mayoría de los casos dependiendo del tipo histológico y del momento en que se realice su diagnóstico ${ }^{(1)}$.

El trasplante de islotes pancreáticos como vemos en el trabajo realizado por Echeverri y colaboradores, constituye una opción terapéutica prometedora para algunos pacientes diabéticos tipo 1 con historia de hipoglicemia frecuentemente no percibida y labilidad glicémica severa ${ }^{(2,3)}$. La diabetes mellitus continúa siendo una entidad de mucho impacto en el mundo entero (más del 10\% de la inversión en salud está relacionada con esta patología). Hace ya más de 100 años se realizó el primer trasplante de islotes por parte del doctor Watson Williamson y el primero con éxito fue solo en 1980. Gracias al progreso en la técnica quirúrgica y en la terapia de inmunosupresión ha sido posible evitar el uso de insulina durante periodos importantes ${ }^{(5)}$. Las premisas y protocolos experimentales de los años veinte en Canadá, realizados por Banting y Best, están tomando un rumbo prometedor y, por qué no decirlo, salvador para este tipo de pacientes. El caso descrito por este grupo de investigadores en el cual se realizó un trasplante de islotes procedente de un donante cadavérico aunque no logró independencia total de la insulina, sí condujo a una reducción significativa de sus cifras metabólicas y a una disminución de los episodios de hipoglicemia. Tenemos que felicitar a este grupo de investigación por estos resultados y estimularlos para que continúen con sus investigaciones, las cuales redundarán, por supuesto, en una mejoría de la calidad de vida de todos nuestros pacientes.

\section{Enrique Ardila \\ Editor}

Revista Colombiana de Endocrinología, Diabetes y Metabolismo

\section{Referencias}

1. Rojas JC., Niño DC., Wandurraga EA, García ME., Camacho PA., Roa JA., et al. Descripción de los hallazgos histopatológicos en la terapia inicial del cáncer de tiroides en un centro de referencia en Bucaramanga, Colombia. Rev ACE Vol(2);1:40-43.

2. Sabogal E., Casas LA., Arango LG., Feriz K., Guzmán G., Gutiérrez 0., et al . Presente y futuro del trasplante de islotes pancreáticos. Un tratamiento innovador para la diabetes tipo I. Rev ACE Vol(2);1:20-32.

3. Echeverri G., Sabogal A., Casas LA., Mesa L., Durán CE., Schweineberg J. et al. Primer tras- plante de islotes realizado en Colombia. Experiencia Fundación Valle del Lilli. Rev ACE Vol(2);1:33-39.

4. Ramírez TA., Sanabria AE. Cirugía para el cáncer papilar de tiroides. Tratado de tiroides. Cap. 49 pp. 348-356. Asociación Colombiana de Endocrinología, Diabetes y Metabolismo. Producciones Científicas Ltda. Bogotá, 2014.

5. Pepper AR., Gala-Lopez B., Ziff 0., Shapiro AJ. Current status of clinical islet trasplantation. World J. Transplant 2013.3(4): 48-53. 